\title{
Research Article \\ Comparing Numerical Methods for Solving Time-Fractional Reaction-Diffusion Equations
}

\author{
Veyis Turut $^{\mathbf{1}}$ and Nuran Güzel ${ }^{2}$ \\ ${ }^{1}$ Department of Mathematics, Faculty of Arts and Sciences, Batman University, 72100 Batman, Turkey \\ ${ }^{2}$ Department of Mathematics, Faculty of Arts and Sciences, Yıldiz Technical University, 34220 İstanbul, \\ Turkey \\ Correspondence should be addressed to Nuran Güzel, nguzel@yildiz.edu.tr
}

Received 7 March 2012; Accepted 29 April 2012

Academic Editors: G. Schimperna and W. Shen

Copyright (C) 2012 V. Turut and N. Güzel. This is an open access article distributed under the Creative Commons Attribution License, which permits unrestricted use, distribution, and reproduction in any medium, provided the original work is properly cited.

\begin{abstract}
Multivariate Padé approximation (MPA) is applied to numerically approximate the solutions of time-fractional reaction-diffusion equations, and the numerical results are compared with solutions obtained by the generalized differential transform method (GDTM). The fractional derivatives are described in the Caputo sense. Two illustrative examples are given to demonstrate the effectiveness of the multivariate Pade approximation (MPA). The results reveal that the multivariate Padé approximation (MPA) is very effective and convenient for solving timefractional reaction-diffusion equations.
\end{abstract}

\section{Introduction}

The fractional calculus and fractional differential equations have recently become increasingly important topics in the literature of engineering, science, and applied mathematics. Application areas include viscoelasticity, electromagnetics, heat conduction, control theory, and diffusion [1-4]. Reaction-diffusion equations are commonly used to model the growth and spreading of biological species. A fractional reaction-diffusion equation (FRDE) can be derived from a continuous-time random walk model when the transport is dispersive [5] or a continuous-time random walk model with temporal memory and sources [6]. The topic has received a great deal of attention recently, for example, in systems biology [7], chemistry, and biochemistry applications [8].

One of the time-fractional reaction-diffusion equations is the time-fractional Fisher equation. It was originally proposed by Fisher [9] as a model for the spatial and temporal propagation of a virile gene in an infinite medium. It is encountered in chemical kinetics [10], flame propagation [11], autocatalytic chemical reaction [12], nuclear reactor theory [13], neurophysiology [14], and branching Brownian motion process [15]. 
Another time-fractional reaction-diffusion equation is the time-fractional FitzhughNagumo equation. It is an important nonlinear reaction-diffusion equation and usually used to model the transmission of nerve impulses $[16,17]$; it is also used in circuit theory, biology, and the area of population genetics [18] as mathematical models.

The generalized differential transform method (GDTM) was presented by [19-21]. This method is based on differential transform method (DTM) [22-25]; the DTM introduces a promising approach for many applications in various domains of science. By using the DTM, a truncated series solution is obtained. This series solution does not exhibit the real behaviors of the problem but gives a good approximation to the true solution in a very small region. Odibat et al. [26] proposed a reliable algorithm of the DTM. The new algorithm accelerates the convergence of the series solution over a large region and improves the accuracy of the DTM. The validity of the modified technique is varied through illustrative examples of LotkaVolterra, Chen, and Lorenz systems. The generalized differential transform method (GDTM) has been applied to differential equations of fractional order in [19-21,27].

In the literature, the univariate Padé approximation has been used to obtain approximate solutions of fractional order $[28,29]$. So the objective of the this paper is to show the application of the multivariate Padé approximation (MPA) to provide approximate solutions for time-fractional diffusion-reaction equations and to make comparison with the generalized differential transform method (GDTM).

\section{Multivariate Padé Approximation}

The principles and theory of the multivariate Padé approximation and its applicability for various of differential equations are given in [30-40]. Consider the bivariate function $f(x, y)$ with Taylor series development

$$
f(x, y)=\sum_{i, j=0}^{\infty} c_{i j} x^{i} y^{j}
$$

around the origin. We know that a solution of univariate Padé approximation problem for

$$
f(x)=\sum_{i=0}^{\infty} c_{i} x^{i}
$$

is given by

$$
\begin{gathered}
p(x)=\left|\begin{array}{cccc}
\sum_{i=0}^{m} c_{i} x^{i} & x \sum_{i=0}^{m-1} c_{i} x^{i} & \cdots & x^{n} \sum_{i=0}^{m-n} c_{i} x^{i} \\
c_{m+1} & c_{m} & \cdots & c_{m+1-n} \\
\vdots & \vdots & \ddots & \vdots \\
c_{m+n} & c_{m+n-1} & \cdots & c_{m}
\end{array}\right| \\
q(x)=\left|\begin{array}{cccc}
1 & x & \cdots & x^{n} \\
c_{m+1} & c_{m} & \cdots & c_{m+1-n} \\
\vdots & \vdots & \ddots & \vdots \\
c_{m+n} & c_{m+n-1} & \cdots & c_{m}
\end{array}\right|
\end{gathered}
$$


Let us now multiply $j$ th row in $p(x)$ and $q(x)$ by $x^{j+m-1}(j=2, \ldots, n+1)$ and afterwards divide $j$ th column in $p(x)$ and $q(x)$ by $x^{j-1}(j=2, \ldots, n+1)$. This results in a multiplication of numerator and denominator by $x^{m n}$. Having done so, we get

$$
\frac{p(x)}{q(x)}=\frac{\left|\begin{array}{cccc}
\sum_{i=0}^{m} c_{i} x^{i} & \sum_{i=0}^{m-1} c_{i} x^{i} & \cdots & \sum_{i=0}^{m-n} c_{i} x^{i} \\
c_{m+1} x^{m+1} & c_{m} x^{m} & \cdots & c_{m+1-n} x^{m+1-n} \\
\vdots & \vdots & \ddots & \vdots \\
c_{m+n} x^{m+n} & c_{m+n-1} x^{m+n-1} & \cdots & c_{m} x^{m}
\end{array}\right|}{\begin{array}{cccc}
1 & \cdots & 1 \\
c_{m+1} x^{m+1} & c_{m} x^{m} & \cdots & c_{m+1-n} x^{m+1-n} \\
\vdots & \vdots & \ddots & \vdots \\
c_{m+n} x^{m+n} & c_{m+n-1} x^{m+n-1} & \cdots & c_{m} x^{m}
\end{array} \mid}
$$

if $\left(D=\operatorname{det} D_{m, n} \neq 0\right)$.

This quotient of determinants can also immediately be written down for a bivariate function $f(x, y)$. The sum $\sum_{i=0}^{k} c_{i} x^{i}$ will be replaced with $k$ th partial sum of the Taylor series development of $f(x, y)$ and the expression $c_{k} x^{k}$ by an expression that contains all the terms of degree $k$ in $f(x, y)$. Hereby, a bivariate term $c_{i j} x^{i} y^{j}$ is said to be of degree $i+j$. If we define

$$
\begin{aligned}
p(x, y) & =\left|\begin{array}{cccc}
\sum_{i+j=0}^{m} c_{i j} x^{i} y^{j} & \sum_{i+j=0}^{m-1} c_{i j} x^{i} y^{j} & \ldots & \sum_{i+j=0}^{m-n} c_{i j} x^{i} y^{j} \\
\sum_{i+j=m+1} c_{i j} x^{i} y^{j} & \sum_{i+j=m} c_{i j} x^{i} y^{j} & \ldots & \sum_{i+j=m+1-n} c_{i j} x^{i} y^{j} \\
\vdots & \vdots & \ddots & \vdots \\
\sum_{i+j=m+n} c_{i j} x^{i} y^{j} & \sum_{i+j=m+n-1} c_{i j} x^{i} y^{j} & \cdots & \sum_{i+j=m} c_{i j} x^{i} y^{j}
\end{array}\right|, \\
q(x, y) & =\left|\begin{array}{cccc}
1 & 1 & \ldots & 1 \\
\sum_{i+j=m+1} c_{i j} x^{i} y^{j} & \sum_{i+j=m} c_{i j} x^{i} y^{j} & \cdots & \sum_{i+j=m+1-n} c_{i j} x^{i} y^{j} \\
\vdots & \vdots & \ddots & \vdots \\
\sum_{i+j=m+n} c_{i j} x^{i} y^{j} & \sum_{i+j=m+n-1} c_{i j} x^{i} y^{j} & \cdots & \sum_{i+j=m} c_{i j} x^{i} y^{j}
\end{array}\right|,
\end{aligned}
$$

then it is easy to see that $p(x, y)$ and $q(x, y)$ are of the form

$$
p(x, y)=\sum_{i+j=m n}^{m n+m} a_{i j} x^{i} y^{j}, \quad q(x, y)=\sum_{i+j=m n}^{m n+n} b_{i j} x^{i} y^{j}
$$

We know that $p(x, y)$ and $q(x, y)$ are called Padé equations [30]. So the multivariate Padé approximant of order $(m, n)$ for $f(x, y)$ is defined as,

$$
r_{m, n}(x, y)=\frac{p(x, y)}{q(x, y)}
$$




\section{Generalized Differential Transform Method}

The fractional derivatives are described in the Caputo sense which are defined in [41] as

$$
D^{\alpha} f(x)=J^{m-\alpha} D^{m} f(x)=\frac{1}{\Gamma(m-\alpha)} \int_{0}^{x}(x-t)^{m-\alpha-1} f^{m}(t) d t,
$$

for $m-1<\alpha \leq m, m \in \mathbb{N}, x>0$; for $m$ to be the smallest integer that exceeds $\alpha$, the Caputo time-fractional derivative operator of order $\alpha>0$ is defined as

$$
D_{t}^{\alpha} u(x, t)=\frac{\partial^{\alpha} u(x, t)}{\partial t^{\alpha}}= \begin{cases}\frac{1}{\Gamma(m-\alpha)} \int_{0}^{t}(t-\tau)^{m-\alpha-1} \frac{\partial^{m} u(x, \tau)}{\partial \tau^{m}} d \tau, & \text { for } m-1<\alpha<m, \\ \frac{\partial^{m} u(x, t)}{\partial t^{m}}, & \text { for } \alpha=m \in \mathbb{N} .\end{cases}
$$

The basic definitions and fundamental operations of generalized differential transform method are defined in [19-21] as follows.

Definition 3.1. The generalized differential transform of the function $u(x, y)$ is given as follows:

$$
U_{\alpha, \beta}(k, h)=\frac{1}{\Gamma(\alpha k+1) \Gamma(\beta h+1)}\left[\left(D_{x_{0}}^{\alpha}\right)^{k}\left(D_{y_{0}}^{\beta}\right)^{h}\right]_{\left(x_{0}, y_{0}\right)},
$$

where $\left(D_{x_{0}}^{\alpha}\right)^{k}=D_{x_{0}}^{\alpha} \cdot D_{x_{0}}^{\alpha} \cdots D_{x_{0}}^{\alpha}$.

Definition 3.2. The generalized differential inverse transform of $U_{\alpha, \beta}(k, h)$ is defined as follows:

$$
u(x, y)=\sum_{k=0}^{\infty} \sum_{h=0}^{\infty} U_{\alpha, \beta}(k, h)\left(x-x_{0}\right)^{k \alpha}\left(y-y_{0}\right)^{h \beta} .
$$

The fundamental operations of generalized differential transform method are listed in Table 1 (see [19-21]).

\section{Numerical Experiments}

In this section, two methods, GDTM and MPA, will be illustrated by two examples, the time-fractional Fisher equation and the time-fractional Fitzhugh-Nagumo equation. All the numerical results are calculated by using the software Maple12. The general model of reaction-diffusion equations is

$$
\frac{\partial^{\alpha} u}{\partial t^{\alpha}}=D \frac{\partial^{2} u}{\partial x^{2}}+f(u), \quad 0<\alpha \leq 1, t>0, x \in \mathfrak{R},
$$

where $D$ is the diffusion coefficient, and $f(u)$ is a nonlinear function representing reaction kinetics. 


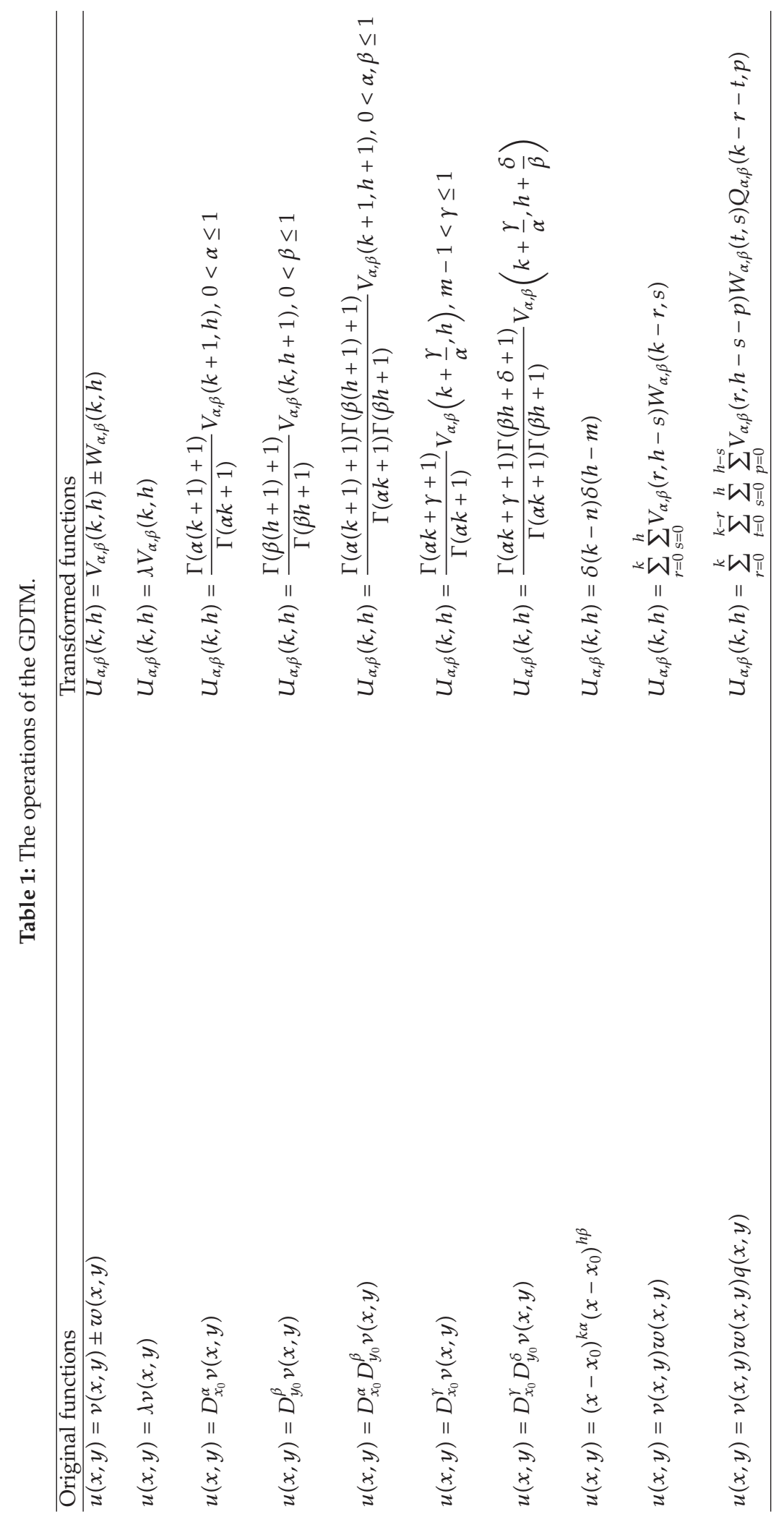


Example 4.1. Let us consider (4.1) with $f(u)=6 u(1-u)$, then we have the time-fractional Fisher equation [27]

$$
D_{t}^{\alpha} u=D_{x}^{2} u+6 u(1-u), \quad 0<\alpha \leq 1, t>0, x \in \mathfrak{R},
$$

subject to the initial condition

$$
u(x, 0)=\frac{1}{\left(1+e^{x}\right)^{2}} .
$$

Selecting $\beta=1$ and applying the generalized differential transform of (4.2), using the related definitions in Table 1, Rida et al. [27] solved as it follows:

$$
\begin{aligned}
& \frac{\Gamma(\alpha(h+1)+1)}{\Gamma(\alpha h+1)} U_{\alpha, 1}(k, h+1) \\
& \quad=(k+1)(k+2) U_{\alpha, 1}(k+2, h)+6 U_{\alpha, 1}(k, h)-6 \sum_{r=0}^{k} \sum_{s=0}^{h} U_{\alpha, 1}(r, h-s) U_{\alpha, 1}(k-r, s),
\end{aligned}
$$

that is,

$$
\begin{aligned}
& U_{\alpha, 1}(k, h+1) \\
& \quad=\frac{\Gamma(\alpha(h+1)+1)}{\Gamma(\alpha h+1)}\left[(k+1)(k+2) U_{\alpha, 1}(k+2, h)+6 U_{\alpha, 1}(k, h)-6 \sum_{r=0}^{k} \sum_{s=0}^{h} U_{\alpha, 1}(r, h-s) U_{\alpha, 1}(k-r, s)\right] .
\end{aligned}
$$

By equating the series form of (4.3) with (3.4), the initial transformation coefficients $U_{\alpha, 1}(k, 0)$, $k=0,1,2, \ldots$ can be obtained as follows:

$$
\begin{gathered}
U_{\alpha, 1}(0,0)=\frac{1}{4}, \quad U_{\alpha, 1}(1,0)=-\frac{1}{4}, \quad U_{\alpha, 1}(2,0)=\frac{1}{16}, \\
U_{\alpha, 1}(3,0)=\frac{1}{48}, \quad U_{\alpha, 1}(4,0)=-\frac{1}{96} .
\end{gathered}
$$

By applying (4.6) into (4.5), some values of $U_{\alpha, 1}(k, h)$ can be obtained as given in Table 1 . Consequent substitution of all $U_{\alpha, 1}(k, h)$ into (3.4) and after some manipulations, the series from solutions of (4.2) and (4.3) has been obtained in [27] as

$$
\begin{aligned}
u(x, t)= & \left(\frac{1}{4}+\frac{5}{4 \Gamma(\alpha+1)} t^{\alpha}+\frac{25}{8 \Gamma(2 \alpha+1)} t^{2 \alpha}+\cdots\right) \\
& +\left(-\frac{1}{4}-\frac{5}{8 \Gamma(\alpha+1)} t^{\alpha}+\frac{25}{8 \Gamma(2 \alpha+1)} t^{2 \alpha}+\cdots\right) x \\
& +\left(\frac{1}{16}-\frac{5}{16 \Gamma(\alpha+1)} t^{\alpha}-\frac{25}{8 \Gamma(2 \alpha+1)} t^{2 \alpha}+\cdots\right) x^{2} \\
& +\left(\frac{1}{48}-\frac{5}{24 \Gamma(\alpha+1)} t^{\alpha}-\frac{25}{24 \Gamma(2 \alpha+1)} t^{2 \alpha}+\cdots\right) x^{3} \\
& +\left(-\frac{1}{96}+\frac{5}{96 \Gamma(\alpha+1)} t^{\alpha}+\frac{425}{384 \Gamma(2 \alpha+1)} t^{2 \alpha}+\cdots\right) x^{4} .
\end{aligned}
$$


$u(x, t)$ can be written in the form:

$$
\begin{aligned}
u(x, t)= & \left(\frac{1}{4}-\frac{1}{4} x+\frac{1}{48} x^{3}-\frac{1}{96} x^{4}+\cdots\right)+\left(\frac{5}{4}-\frac{5}{8} x-\frac{5}{16} x^{2}-\frac{5}{24} x^{3}+\frac{5}{96} x^{4}+\cdots\right) \frac{t^{\alpha}}{\Gamma(\alpha+1)} \\
& +\left(\frac{25}{8}+\frac{25}{8} x-\frac{25}{8} x^{2}-\frac{25}{24} x^{3}+\frac{425}{384} x^{4}+\cdots\right) \frac{t^{2 \alpha}}{\Gamma(2 \alpha+1)}
\end{aligned}
$$

The exact solution of (4.2), for the special case $\alpha=1.0$, is given in [27] as

$$
u(x, t)=\frac{1}{\left(1+e^{x-5 t}\right)^{2}} .
$$

We have the generalized differential transform method solution for the time-fractional Fisher equation $(4.2)$ (when $=1.0)$ as

$$
\begin{gathered}
u(x, t)=0.2500000000-0.2500000000 x-0.06250000000 x^{2}+0.02083333333 x^{3} \\
-0.01041666667 x^{4}+\left(1.250000000-0.6250000000 x-0.3125000000 x^{2}\right. \\
\left.-0.2083333333 x^{3}+0.05208333333 x^{4}\right) t \\
+0.5000000000\left(3.125000000+3.125000000 x-3.125000000 x^{2}\right. \\
\left.-1.401666667 x^{3}+1.106770833 x^{4}\right) t^{2}, \\
=0.2500000000-0.2500000000 x-0.06250000000 x^{2}+0.02083333333 x^{3} \\
-0.01041666667 x^{4}+1.250000000 t-0.6250000000 x t-0.3125000000 x^{2} t \\
-0.2083333333 x^{3} t+0.05208333333 x^{4} t+1.562500000 t^{2}+1.562500000 x t^{2} \\
-1.562500000 x^{2} t^{2}-0.5208333335 x^{3} t^{2}+0.5533854165 x^{4} t^{2}
\end{gathered}
$$

and let

$$
\begin{aligned}
A= & 0.2500000000-0.2500000000 x-0.06250000000 x^{2}+0.02083333333 x^{3} \\
& -0.01041666667 x^{4}+1.250000000 t-0.6250000000 x t-0.3125000000 x^{2} t \\
& -0.2083333333 x^{3} t+1.562500000 t^{2}+1.562500000 x t^{2}-1.562500000 x^{2} t^{2}, \\
B= & 0.2500000000-0.2500000000 x-0.06250000000 x^{2}+0.02083333333 x^{3} \\
& +1.250000000 t-0.6250000000 x t-0.3125000000 x^{2} t+1.562500000 t^{2} \\
& +1.562500000 x t^{2},
\end{aligned}
$$




$$
\begin{aligned}
C= & 0.2500000000-0.2500000000 x-0.06250000000 x^{2} \\
& +1.250000000 t-0.6250000000 x t+1.562500000 t^{2} .
\end{aligned}
$$

Then let us calculate the approximate solution of (4.10) for $m=4$ and $n=2$ by using multivariate Padé approximation. To obtain multivariate Padé equations of (4.10) for $m=4$ and $n=2$, we use (2.5). By using (2.5), we obtain

$$
\begin{aligned}
& p(x, t) \\
& =\left|\begin{array}{ccc}
A & B & C \\
0.05208333333 x^{4} t-0.5208333335 x^{3} t^{2} & A & B \\
0.5533854165 x^{4} t^{2} & 0.05208333333 x^{4} t-0.5208333335 x^{3} t^{2} & A
\end{array}\right| \\
& =-0.5533854165 x^{4}\left(-0.0001225490198 x^{5} t-0.03063725491 t^{5} x^{4}\right. \\
& +0.3082873774 t^{4} x^{3}+0.02037377449 t^{3} x^{4}+0.003604983663 t^{2} x^{10} \\
& +0.00004901960791 x^{5}-0.00001225490198 x^{6}-1.470588235 t^{4} \\
& -0.3676470590 t^{4} x^{2}+0.002757352939 t^{3} x^{6}-0.04289215684 t^{3} x^{3} \\
& +0.09803921566 t^{3} x^{2}-0.0001633986928 t x^{6}-0.2573529412 t^{3} x \\
& +0.1608455885 t^{4} x+0.0001225490196 x^{7} t^{2}-0.01072303921 x^{5} t^{4} \\
& -0.06318933824 t^{5} x^{5}-0.001914828416 t^{5} x^{6}+0.00004084967320 x^{8} t^{2} \\
& -0.002323325162 x^{7} t^{3}+0.02221200981 x^{6} t^{4}-0.00002042483661 x^{9} t^{2} \\
& -0.0006382761434 x^{8} t^{3}+0.007531658495 x^{7} t^{4} \\
& -0.4084967326 \times 10^{-5} x^{7}+0.2042483663 \times 10^{-5} x^{8}-9.191176472 t^{6} \\
& +5.840226718 t^{6} x^{2}-9.334788603 t^{6} x-7.352941178 t^{5} \\
& +4.049862133 t^{5} x^{3}-2.202052696 t^{5} x^{2}+1.953125001 t^{5} x \\
& +0.3498391544 t^{4} x^{4}+0.05895118467 t^{3} x^{5}+0.002024611930 t^{2} x^{6} \\
& +0.0001021241832 t x^{7}-0.00004901960791 x^{4}-0.006587009808 t^{2} x^{4} \\
& +0.02205882354 t^{2} x^{3}+0.001470588237 t x^{4}-0.03431372552 t^{2} x^{2} \\
& \left.-0.001960784315 t x^{3}\right),
\end{aligned}
$$


ISRN Mathematical Analysis

$$
\begin{aligned}
q(x, t)=\left|\begin{array}{ccc}
1 & 1 & 1 \\
0.05208333333 x^{4} t-0.5208333335 x^{3} t^{2} & A & B \\
0.5533854165 x^{4} t^{2} & 0.05208333333 x^{4} t-0.5208333335 x^{3} t^{2} & A
\end{array}\right| \\
=0.5533854165 x^{4}\left(1.029411764 t^{3} x+5.882352942 t^{4}+0.091911764 t^{4} x\right. \\
-0.001960784314 t^{2} x^{5}+0.001960784314 t^{3} x^{4}+0.02083333333 t^{3} x^{5} \\
+0.01531862746 t^{2} x^{4}+0.1102941176 t^{3} x^{3}+2.052696079 t^{4} x^{2} \\
+0.007843137258 t x^{3}+0.1372549020 t^{2} x^{2}+0.009803921564 t^{2} x^{3} \\
\\
\left.-0.0490196080 t^{3} x^{2}+0.0001960784315 x^{4}+0.0009803921572 t x^{4}\right),
\end{aligned}
$$

where $\mathcal{A}$ denotes $-0.01041666667 x^{4}-0.2083333333 x^{3} t-1.562500000 x^{2} t^{2}$, and $B$ denotes $0.02083333333 x^{3}-0.3125000000 x^{2} t+1.562500000 x t^{2}$. So the multivariate Padé approximation is of order $(4,2)$ for $(4.10)$, that is,

$$
\begin{aligned}
{[4,2]_{(x, t)}=-(} & -0.0001225490198 x^{5} t-0.03063725491 t^{5} x^{4} \\
& +0.3082873774 t^{4} x^{3}+0.02037377449 t^{3} x^{4}+0.003604983663 t^{2} x^{5} \\
& +0.00004901960791 x^{5}-0.00001225490198 x^{6}-1.470588235 t^{4} \\
& -0.3676470590 t^{4} x^{2}+0.002757352939 t^{3} x^{6}-0.04289215684 t^{3} x^{3} \\
& +0.09803921566 t^{3} x^{2}-0.0001633986928 t x^{6}-0.2573529412 t^{3} x \\
& +0.1608455885 t^{4} x+0.0001225490196 x^{7} t^{2}-0.01072303921 x^{5} t^{4} \\
& -0.06318933824 t^{5} x^{5}-0.001914828416 t^{5} x^{6}+0.00004084967320 x^{8} t^{2} \\
& -0.002323325162 x^{7} t^{3}+0.02221200981 x^{6} t^{4}-0.00002042483661 x^{9} t^{2} \\
& -0.0006382761434 x^{8} t^{3}+0.007531658495 x^{7} t^{4}-0.4084967326 \times 10^{-5} x^{7} \\
& +0.2042483663 \times 10^{-5} x^{8}-9.191176472 t^{6}+5.840226718 t^{6} x^{2}-9.334788603 t^{6} x \\
& -7.352941178 t^{5}+4.049862133 t^{5} x^{3}-2.202052696 t^{5} x^{2}+1.953125001 t^{5} x \\
& +0.3498391544 t^{4} x^{4}+0.05895118467 t^{3} x^{5}+0.002024611930 t^{2} x^{6} \\
& +0.0001021241832 t x^{7}-0.00004901960791 x^{4}-0.006587009808 t^{2} x^{4} \\
& +0.02205882354 t^{2} x^{3}+0.001470588237 t x^{4}-0.03431372552 t^{2} x^{2} \\
& \left.-0.001960784315 t x^{3}\right) \\
& /\left(1.029411764 t^{3} x+5.882352942 t^{4}+0.091911764 t^{4} x\right.
\end{aligned}
$$




$$
\begin{aligned}
& -0.001960784314 t^{2} x^{5}+0.001960784314 t^{3} x^{4}+0.02083333333 t^{3} x^{5} \\
& +0.01531862746 t^{2} x^{4}+0.1102941176 t^{3} x^{3}+2.052696079 t^{4} x^{2} \\
& +0.007843137258 t x^{3}+0.1372549020 t^{2} x^{2}+0.009803921564 t^{2} x^{3} \\
& \left.-0.0490196080 t^{3} x^{2}+0.0001960784315 x^{4}+0.0009803921572 t x^{4}\right) .
\end{aligned}
$$

The generalized differential transform method gives the solution for the time-fractional Fisher equation (4.2) (when $\alpha=0.5$ ) which is given by

$$
\begin{aligned}
u(x, t)= & 0.2500000000-0.2500000000 x-0.06250000000 x^{2}+0.02083333333 x^{3}-0.01041666667 x^{4} \\
+ & 1.128379167(1.250000000-0.6250000000 x \\
& \left.\quad-0.3125000000 x^{2}-0.20833333333 x^{3}+0.05208333333 x^{4}\right) t^{0.5} \\
& +\left(3.125000000+3.125000000 x-3.125000000 x^{2}-1.401666667 x^{3}+1.106770833 x^{4}\right) t .
\end{aligned}
$$

For simplicity, let $t^{1 / 2}=a$, then

$$
\begin{aligned}
u(x, t)= & 0.2500000000-0.2500000000 x-0.06250000000 x^{2} \\
+ & 0.02083333333 x^{3}-0.01041666667 x^{4} \\
+ & 1.128379167(1.250000000-0.6250000000 x \\
& \left.\quad-0.3125000000 x^{2}-0.2083333333 x^{3}+0.05208333333 x^{4}\right) a \\
& +\left(3.125000000+3.125000000 x-3.125000000 x^{2}-1.401666667 x^{3}\right. \\
& \left.\quad+1.106770833 x^{4}\right) a^{2}, \\
= & 0.2500000000-0.2500000000 x-0.06250000000 x^{2}+0.02083333333 x^{3} \\
& -0.01041666667 x^{4}+1.410473959 a-0.7052369794 a x-0.3526184897 a x^{2} \\
& -0.2350789931 a x^{3}+0.05876974828 a x^{4}+3.125000000 a^{2}+3.125000000 a^{2} x \\
& -3.125000000 a^{2} x^{2}-1.401666667 a^{2} x^{3}+1.106770833 a^{2} x^{4},
\end{aligned}
$$

and let

$$
\begin{aligned}
E= & 0.2500000000-0.2500000000 x-0.06250000000 x^{2}+0.02083333333 x^{3} \\
& -0.01041666667 x^{4}+1.410473959 a-0.7052369794 a x-0.3526184897 a x^{2} \\
& -0.2350789931 a x^{3}+3.125000000 a^{2}+3.125000000 a^{2} x-3.125000000 a^{2} x^{2},
\end{aligned}
$$


ISRN Mathematical Analysis

$$
\begin{aligned}
F= & 0.2500000000-0.2500000000 x-0.06250000000 x^{2}+0.02083333333 x^{3} \\
& +1.410473959 a-0.7052369794 a x-0.3526184897 a x^{2}+3.125000000 a^{2} \\
& +3.125000000 a^{2} x \\
G= & 0.2500000000-0.2500000000 x-0.06250000000 x^{2} \\
& +1.410473959 a-0.7052369794 a x+3.125000000 a^{2} .
\end{aligned}
$$

Then, using (2.5) to calculate the multivariate Padé equations for (4.16), we get

$$
\begin{aligned}
& p(x, a) \\
& =\left|\begin{array}{ccc}
E & F & G \\
0.05876974828 a x^{4}-1.401666667 a^{2} x^{3} & \mathcal{C} & \Phi \\
1.106770833 a^{2} x^{4} & 0.05876974828 a x^{4}-1.401666667 a^{2} x^{3} & \mathcal{C}
\end{array}\right| \\
& =-1.106770833 x^{4}\left(1.724963655 a^{4} x+0.00055331270431 a x^{4}\right. \\
& +0.00002450980395 x^{5}-0.6127450986 \times 10^{-5} x^{6}-0.2042483661 \times 10^{-5} x^{7} \\
& +0.1021241831 \times 10^{-5} x^{8}+0.02621254569 a^{2} x^{3}-0.005445232711 a^{2} x^{4} \\
& -0.03677161152 a^{2} x^{2}-0.0008296905640 a x^{3}-0.2074226410 a^{3} x \\
& +0.000104497505 a^{2} x^{5}+0.07657717919 a^{3} x^{4}+0.0659779402 a^{4} x^{3} \\
& -0.1247554646 a^{3} x^{3}-1.014826625 a^{4} x^{2}+0.1 \times 10^{-12} x^{5} a \\
& +0.1019438376 a^{3} x^{2}-0.00006914088034 a x^{6}-2.941176471 a^{4} \\
& -36.76470589 a^{6}+23.36090687 a^{6} x^{2}-37.33915441 a^{6} x \\
& -16.59381128 a^{5}+9.204379692 a^{5} x^{3}-2.830454788 a^{5} x^{2} \\
& +5.444844332 a^{5} x+0.3449658071 a^{4} x^{4}+0.07042023202 a^{3} x^{5} \\
& \left.+0.001477816147 a^{2} x^{6}+0.00004609392028 a x^{7}-0.00002450980395 x^{4}\right) \text {. } \\
& q(x, a)
\end{aligned}
$$

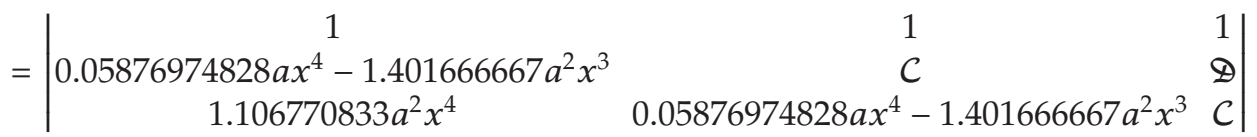

$$
\begin{aligned}
& =1.106770833 x^{4}\left(11.76470588 a^{4}+0.003318762259 a x^{3}\right. \\
& +0.00009803921577 x^{4}+0.1470864461 a^{2} x^{2}+0.02351215238 a^{2} x^{3} \\
& +0.01353735183 a^{2} x^{4}+0.1244535845 a^{3} x^{3}+4.105392158 a^{4} x^{2} \\
& -0.4079311941 a^{3} x^{2}+0.183823528 a^{4} x+0.0005531270429 a x^{4} \\
& \left.+0.8296905634 a^{3} x\right) \text {. }
\end{aligned}
$$


where $\mathcal{C}$ is $-0.01041666667 x^{4}-0.2350789931 a x^{3}-3.125000000 a^{2} x^{2}$, and $\Phi$ is $0.02083333333 x^{3}-$ $0.3526184897 a x^{2}-0.3125000000 a^{2} x$ recalling that $t^{1 / 2}=a$, we get multivariate Padé approximation of order $(4,2)$ for $(4.15)$, that is,

$$
\begin{aligned}
{[4,2]_{(x, t)}=- } & 1.724963655 t^{2} x+0.00055331270431 \sqrt{t} x^{4}+0.00002450980395 x^{5} \\
- & 0.6127450986 \times 10^{-5} x^{6}-0.2042483661 \times 10^{-5} x^{7}+0.1021241831 \times 10^{-5} x^{8} \\
+ & 0.02621254569 t x^{3}-0.005445232711 t x^{4}-0.03677161152 t x^{2} \\
- & 0.0008296905640 \sqrt{t} x^{3}-0.2074226410 t^{3 / 2} x+0.000104497505 t x^{5} \\
+ & 0.07657717919 t^{3 / 2} x^{4}+0.0659779402 t^{2} x^{3}-0.1247554646 t^{3 / 2} x^{3} \\
- & 1.014826625 t^{2} x^{2}+0.1 \times 10^{-12} x^{5} \sqrt{t}+0.1019438376 t^{3 / 2} x^{2} \\
- & 0.00006914088034 \sqrt{t} x^{6}-2.941176471 t^{2}-36.76470589 t^{3} \\
+ & 23.36090687 t^{3} x^{2}-37.33915441 t^{3} x-16.59381128 t^{5 / 2}+9.204379692 t^{5 / 2} x^{3} \\
- & 2.830454788 t^{5 / 2} x^{2}+5.444844332 t^{5 / 2} x+0.3449658071 t^{2} x^{4} \\
+ & 0.07042023202 t^{3 / 2} x^{5}+0.001477816147 t x^{6} \\
+ & \left.0.00004609392028 \sqrt{t} x^{7}-0.00002450980395 x^{4}\right) \\
& /\left(11.76470588 t^{2}+0.003318762259 \sqrt{t} x^{3}+0.00009803921577 x^{4}\right. \\
& +0.1470864461 t x^{2}+0.02351215238 t x^{3} \\
& +0.01353735183 t x^{4}+0.1244535845 t^{3 / 2} x^{3}+4.105392158 t^{2} x^{2} \\
& -0.4079311941 t^{3 / 2} x^{2}+0.183823528 t^{2} x+0.0005531270429 \sqrt{t} x^{4} \\
& \left.+0.8296905634 t^{3 / 2} x\right) .
\end{aligned}
$$

The generalized differential transform method gives the solution for the time-fractional Fisher equation (4.2) (when $\alpha=0.75$ ) which is given by

$$
\begin{gathered}
u(x, t)=0.2500000000-0.2500000000 x-0.06250000000 x^{2}+0.02083333333 x^{3} \\
-0.01041666667 x^{4}+1.088065252\left(1.250000000-0.6250000000 x-0.3125000000 x^{2}\right. \\
\left.-0.2083333333 x^{3}+0.05208333333 x^{4}\right) t^{0.75} \\
+0.7522527782(3.125000000+3.125000000 x \\
\left.-3.125000000 x^{2}-1.401666667 x^{3}+1.106770833 x^{4}\right) t^{1.50}
\end{gathered}
$$


ISRN Mathematical Analysis

$$
\begin{aligned}
= & 0.2500000000-0.2500000000 x-0.06250000000 x^{2}+0.02083333333 x^{3} \\
& -0.01041666667 x^{4}+1.360081565 t^{0.75}-0.6800407825 t^{0.75} x-0.3400203912 t^{0.75} x^{2} \\
& -0.22668002608 t^{0.75} x^{3}+0.05667006520 t^{0.75} x^{4}+2.350789932 t^{1.50}+2.350789932 t^{1.50} x \\
& -2.350789932 t^{1.50} x^{2}-0.7835966442 t^{1.50} x^{3}+0.8325714340 t^{1.50} x^{4} .
\end{aligned}
$$

For simplicity, let $t^{1 / 4}=a$, then

$$
\begin{aligned}
u(x, a)= & 0.2500000000-0.2500000000 x-0.06250000000 x^{2}+0.02083333333 x^{3} \\
& -0.01041666667 x^{4}+1.360081565 a^{3}-0.6800407825 a^{3} x-0.3400203912 a^{3} x^{2} \\
& -0.22668002608 a^{3} x^{3}+0.05667006520 a^{3} x^{4}+2.350789932 a^{6}+2.350789932 a^{6} x \\
& -2.350789932 a^{6} x^{2}-0.7835966442 a^{6} x^{3}+0.8325714340 a^{6} x^{4},
\end{aligned}
$$

and let

$$
\begin{aligned}
H= & 0.2500000000-0.2500000000 x-0.06250000000 x^{2}+0.02083333333 x^{3} \\
& -0.01041666667 x^{4}+1.360081565 a^{3}-0.6800407825 a^{3} x-0.3400203912 a^{3} x^{2} \\
& -0.22668002608 a^{3} x^{3}+0.05667006520 a^{3} x^{4} 2.350789932 a^{6}+2.350789932 a^{6} x, \\
K= & 0.2500000000-0.2500000000 x-0.06250000000 x^{2}+0.02083333333 x^{3} \\
& -0.01041666667 x^{4}+1.360081565 a^{3}-0.6800407825 a^{3} x-0.3400203912 a^{3} x^{2} \\
& -0.22668002608 a^{3} x^{3}+2.350789932 a^{6}, \\
L= & 0.2500000000-0.2500000000 x-0.06250000000 x^{2}+0.02083333333 x^{3} \\
& -0.01041666667 x^{4}+1.360081565 a^{3}-0.6800407825 a^{3} x-0.3400203912 a^{3} x^{2} .
\end{aligned}
$$

Then, using (2.5) to calculate the multivariate Padé equations for (4.23), we get

$$
\begin{aligned}
p(x, a) & =\left|\begin{array}{ccc}
H & K & L \\
-2.350789932 a^{6} x^{2} & \mathcal{E} & f \\
-0.7835966442 a^{6} x^{3} & -2.350789932 a^{6} x^{2} & \varepsilon
\end{array}\right| \\
= & 1.842071102 x^{2}\left(0.55703900549 a^{6} x^{4}+14.10473959 a^{12}-0.9467234329 a^{6} x^{3}\right. \\
& -0.00001816058365 x^{10}+0.00003632116729 x^{9}+0.0004358540080 x^{6}
\end{aligned}
$$




$$
\begin{aligned}
& -0.0004358540080 x^{7}+0.0001089635019 x^{8}+0.07834715868 a^{3} x^{4} \\
& -0.03616022708 a^{3} x^{3}+1.500000000 a^{6}+0.8750000002 a^{6} x^{2} \\
& -0.9999999998 a^{6} x-0.04520028386 a^{3} x^{5}+2.040122348 a^{9} x^{2} \\
& +18.80631945 a^{12} x+0.0000987995000 a^{3} x^{10}-5.100305867 a^{9} x^{3} \\
& +0.00387786414 a^{3} x^{6}-0.001848360442 a^{3} x^{8}-0.0006463106887 a^{3} x^{9} \\
& +0.005343335889 a^{3} x^{7}-0.05054699319 a^{6} x^{7}+0.0297127611 a^{6} x^{5} \\
& -0.07855231035 a^{6} x^{6}-1.416751630 a^{9} x^{4}-1.360081565 a^{9} x \\
& \left.+8.160489388 a^{9}\right) a^{6} \\
& q(x, a)=\left|\begin{array}{ccc}
1 & 1 & 1 \\
-2.350789932 a^{6} x^{2} & \mathcal{E} & \mathcal{f} \\
-0.7835966442 a^{6} x^{3} & -2.350789932 a^{6} x^{2} & \mathcal{E}
\end{array}\right| \\
& =1.842071102 x^{2}\left(-0.1446409084 a^{3} x^{3}+0.1687477264 a^{3} x^{4}+5.999999998 a^{6}\right. \\
& +1.999999999 a^{6} x+3.999999999 a^{6} x^{2}+0.001743416031 x^{6} \\
& \left.+0.02410681806 a^{3} x^{5}\right) a^{6},
\end{aligned}
$$

where $\mathcal{E}$ denotes $0.05667006520 a^{3} x^{4}+2.350789932 a^{6} x$, and $\mathcal{F}$ denotes $2.350789932 a^{6}-$ $0.22668002608 a^{3} x^{3}$; recalling that $t^{1 / 4}=a$, we get multivariate Padé approximation of order $(7,2)$ for $(4.21)$, that is,

$$
\begin{aligned}
{[7,2]_{(x, t)}=(} & 0.55703900549 t^{3 / 2} x^{4}+14.10473959 t^{3}-0.9467234329 t^{3 / 2} x^{3} \\
& -0.00001816058365 x^{10}+0.00003632116729 x^{9}+0.0004358540080 x^{6} \\
& -0.0004358540080 x^{7}+0.0001089635019 x^{8}+0.07834715868 t^{3 / 4} x^{4} \\
& -0.03616022708 t^{3 / 4} x^{3}+1.500000000 t^{3 / 2}+0.8750000002 t^{3 / 2} x^{2} \\
& -0.9999999998 t^{3 / 2} x-0.04520028386 t^{3 / 4} x^{5}+2.040122348 t^{9 / 4} x^{2} \\
& +18.80631945 t^{3} x+0.0000987995000 t^{3 / 4} x^{10}-5.100305867 t^{9 / 4} x^{3} \\
& +0.00387786414 t^{3 / 4} x^{6}-0.001848360442 t^{3 / 4} x^{8}-0.0006463106887 t^{3 / 4} x^{9} \\
& +0.005343335889 t^{3 / 4} x^{7}-0.05054699319 t^{3 / 2} x^{7}+0.0297127611 t^{3 / 2} x^{5}
\end{aligned}
$$




$$
\begin{aligned}
& -0.07855231035 t^{3 / 2} x^{6}-1.416751630 t^{9 / 4} x^{4}-1.360081565 t^{9 / 4} x \\
& \left.+8.160489388 t^{9 / 4}\right) \\
& \quad /\left(-0.1446409084 t^{3 / 4} x^{3}+0.1687477264 t^{3 / 4} x^{4}\right. \\
& \quad+5.999999998 t^{3 / 2}+1.999999999 t^{3 / 2} x+3.999999999 t^{3 / 2} x^{2} \\
& \left.\quad+0.001743416031 x^{6}+0.02410681806 t^{3 / 4} x^{5}\right) .
\end{aligned}
$$

As it is presented above, we obtained multivariate Padé approximations of the generalized differential transform method solution of the time-fractional Fisher equation (4.2) for values of $\alpha=1.0, \alpha=0.50$, and $\alpha=0.75$. Table 2 shows the approximate solutions for (4.2) obtained for different values of $\alpha$ using the generalized differential transform method (GDTM) and the multivariate padé approximation (MPA). The values of $\alpha=1.0$ are the only case for which we know the exact solution $u(x, t)=1 /\left(1+e^{x-5 t}\right)^{2}$, and the results of multivariate padé approximation (MPA) are in excellent agreement with the exact solution and those obtained by the generalized differential transform method (GDTM).

Example 4.2. Let us consider (4.1) with $f(u)=u(1-u)(u-\mu)$, then we have the time-fractional Fitzhugh-Nagumo equation [27]

$$
D_{t}^{\alpha} u=D_{x}^{2} u+u(1-u)(u-\mu), \quad \mu>0,0<\alpha \leq 1, t>0, x \in \mathfrak{R}
$$

subject to the initial condition

$$
u(x, 0)=\frac{1}{\left(1+e^{-x / \sqrt{2}}\right)}
$$

Taking the generalized differential transform of (4.27), using the related definitions in Table 1, Rida et al. [27] solved it as follows:

$$
\begin{aligned}
& \frac{\Gamma(\alpha(h+1)+1)}{\Gamma(\alpha h+1)} U_{\alpha, 1}(k, h+1) \\
& =(k+1)(k+2) U_{\alpha, 1}(k+2, h)-\mu U_{\alpha, 1}(k, h)+(1+\mu) \sum_{r=0}^{k} \sum_{s=0}^{h} U_{\alpha, 1}(r, h-s) U_{\alpha, 1}(k-r, s) \\
& \quad-\sum_{r=0}^{k} \sum_{t=0}^{k-r} \sum_{s=0}^{h} \sum_{p=0}^{h-s} U_{\alpha, 1}(r, h-s-p) U_{\alpha, 1}(t, s) U_{\alpha, 1}(k-r-t, p)
\end{aligned}
$$




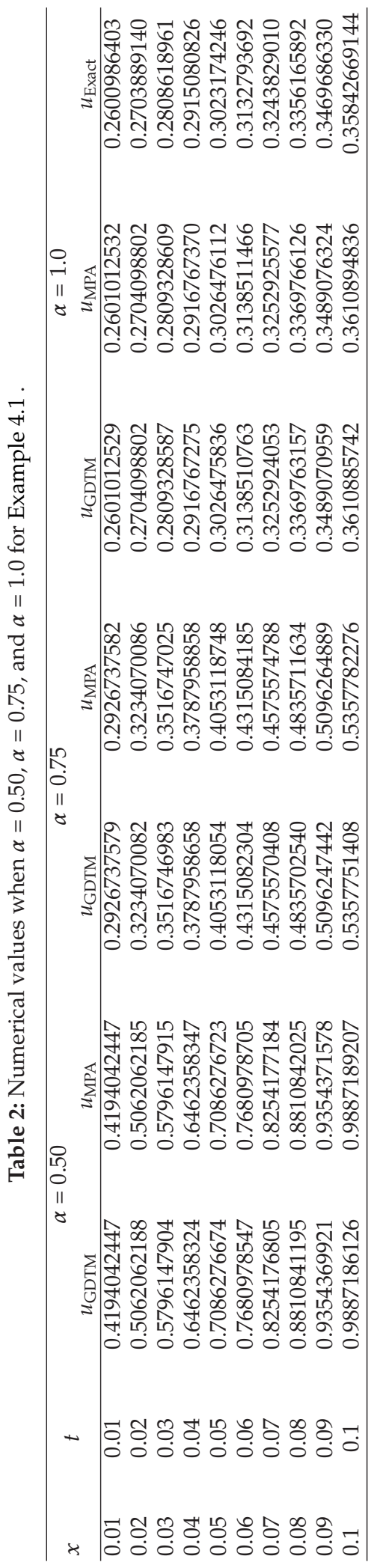


that is,

$$
\begin{aligned}
& U_{\alpha, 1}(k, h+1) \\
& \qquad \frac{\Gamma(\alpha(h+1)+1)}{\Gamma(\alpha h+1)}\left[(k+1)(k+2) U_{\alpha, 1}(k+2, h)-\mu U_{\alpha, 1}(k, h)\right. \\
& +(1+\mu) \sum_{r=0}^{k} \sum_{s=0}^{h} U_{\alpha, 1}(r, h-s) U_{\alpha, 1}(k-r, s) \\
& \left.\quad-\sum_{r=0}^{k} \sum_{t=0}^{k-r} \sum_{s=0}^{h} \sum_{p=0}^{h-s} U_{\alpha, 1}(r, h-s-p) U_{\alpha, 1}(t, s) U_{\alpha, 1}(k-r-t, p)\right] .
\end{aligned}
$$

By equating the series form of (4.28) with (3.4), the initial transformation coefficients $U_{\alpha, 1}(k, 0), k=0,1,2, \ldots$ can be obtained as follows:

$$
\begin{gathered}
U_{\alpha, 1}(0,0)=\frac{1}{2}, \quad U_{\alpha, 1}(1,0)=-\frac{1}{4 \sqrt{2}}, \quad U_{\alpha, 1}(2,0)=0 \\
U_{\alpha, 1}(3,0)=-\frac{1}{96}, \quad U_{\alpha, 1}(4,0)=0
\end{gathered}
$$

By applying (4.31) into (4.30), some values of $U_{\alpha, 1}(k, h)$ can be obtained as given in Table 1 . Consequent substitution of all $U_{\alpha, 1}(k, h)$ into (3.4) and after some manipulations, the series from solutions of (4.27) and (4.28) has been obtained in [27] as:

$$
\begin{aligned}
u(x, t)= & \left(\frac{1}{2}+\frac{1-2 \mu}{8 \Gamma(\alpha+1)} t^{\alpha}+\frac{(1-2 \mu)^{2}}{8 \Gamma(2 \alpha+1)} t^{2 \alpha}+\cdots\right)+\left(-\frac{1}{4 \sqrt{2}}-\frac{(1-2 \mu)^{2}}{32 \sqrt{2} \Gamma(2 \alpha+1)} t^{2 \alpha}+\cdots\right) x \\
& +\left(\frac{1-2 \mu}{64 \Gamma(\alpha+1)} t^{\alpha}+\frac{(1-2 \mu)^{2}}{64 \Gamma(2 \alpha+1)} t^{2 \alpha}+\cdots\right) x^{2}+\left(-\frac{1}{96 \sqrt{2}}+\frac{(1-2 \mu)^{2}}{192 \sqrt{2} \Gamma(2 \alpha+1)} t^{2 \alpha}+\cdots\right) x^{3} \\
& +\left(\frac{1-2 \mu}{768 \Gamma(\alpha+1)} t^{\alpha}-\frac{(1-2 \mu)^{2}}{768 \Gamma(2 \alpha+1)} t^{2 \alpha}+\cdots\right) x^{4}
\end{aligned}
$$

$u(x, t)$ can be written in the form:

$$
\begin{aligned}
u(x, t)= & \left(\frac{1}{2}-\frac{1}{4 \sqrt{2}} x-\frac{1}{96 \sqrt{2}} x^{3}+\frac{1}{1920 \sqrt{2}} x^{5}+\cdots\right) \\
& +\frac{1-2 \mu}{2}\left(\frac{1}{4}-\frac{1}{32} x^{2}+\frac{1}{384} x^{4}-\frac{17}{92160} x^{6}+\cdots\right) \frac{t^{\alpha}}{\Gamma(\alpha+1)} \\
& -\left(\frac{(1-2 \mu)}{2}\right)^{2}\left(\frac{1}{2}+\frac{1}{8 \sqrt{2}} x-\frac{1}{16} x^{2}-\frac{1}{48 \sqrt{2}} x^{3}+\frac{1}{192} x^{4}+\cdots\right) \frac{t^{2 \alpha}}{\Gamma(2 \alpha+1)}
\end{aligned}
$$


The exact solution of (4.27), for the special case $\alpha=1.0$, is given in [27]

$$
u(x, t)=\frac{1}{1+e^{(1 / \sqrt{2})(x+((1-2 \mu) / \sqrt{2}) t)}} .
$$

We have the generalized differential transform method solution for the time-fractional Fitzhugh-Nagumo equation (4.27) (when $\alpha=1.0$ and $\mu=0.45$ ) as

$$
\begin{aligned}
u(x, t)= & 0.5000000000-0.1767766952 x-0.007365695635 x^{3}+0.0003682847818 x^{5} \\
+ & 0.05000000000\left(0.2500000000-0.03125000000 x^{2}+0.002604166667 x^{4}\right. \\
& \left.\quad-0.0001844618056 x^{6}\right) t \\
- & 0.001250000000(0.5000000000-0.08838834762 x \\
& \left.\quad-0.06250000000 x^{2}-0.01473139128 x^{3}+0.005208333333 x^{4}\right) t^{2} \\
= & 0.5000000000-0.1767766952 x-0.007365695635 x^{3} \\
+ & 0.0003682847818 x^{5}+0.01250000000 t-0.001562500000 t x^{2} \\
+ & 0.0001302088884 t x^{4}-0.9223090280 \times 10^{-5} t x^{6}-0.0006250000000 t^{2} \\
+ & 0.0001104854345 t^{2} x+0.00007812500000 t^{2} x^{2}+0.00001841423910 t^{2} x^{3} \\
& -0.6510416666 \times 10^{-5} t^{2} x^{4}
\end{aligned}
$$

and let

$$
\begin{aligned}
M= & 0.5000000000-0.1767766952 x-0.007365695635 x^{3} \\
& +0.0003682847818 x^{5}+0.01250000000 t-0.001562500000 t x^{2} \\
& +0.0001302088884 t x^{4}-0.0006250000000 t^{2}+0.0001104854345 t^{2} x \\
& +0.00007812500000 t^{2} x^{2}+0.00001841423910 t^{2} x^{3}, \\
N= & 0.5000000000-0.1767766952 x-0.007365695635 x^{3} \\
& +0.01250000000 t-0.001562500000 t x^{2}-0.0006250000000 t^{2} \\
& +0.0001104854345 t^{2} x+0.00007812500000 t^{2} x^{2}, \\
R= & 0.5000000000-0.1767766952 x-0.007365695635 x^{3} \\
& +0.01250000000 t-0.001562500000 t x^{2}-0.0006250000000 t^{2} \\
& +0.0001104854345 t^{2} x .
\end{aligned}
$$


Then let us calculate the approximate solution of (4.35) for $m=5$ and $n=2$ by using multivariate Padé approximation. To obtain multivariate Padé equations of (4.35) for $m=5$ and $n=2$, we use (2.5). By using (2.5), We obtain

$$
\begin{aligned}
& p(x, t) \\
& =\left|\begin{array}{ccc}
M & N & R \\
-0.6510416666 \times 10^{-5} t^{2} x^{4} & \mathcal{G} & 0.00007812500000 t^{2} x^{2} \\
-0.9223090280 \times 10^{-5} t x^{6} & -0.6510416666 \times 10^{-5} t^{2} x^{4} & \mathcal{G}
\end{array}\right| \\
& =0.6004616067 \times 10^{-10} x^{6}\left(-399.3073584 x^{5}+1.529411773 t^{2} x^{4}-25.29411764 t x^{6}\right. \\
& -49.91341983 t^{2} x^{3}+254.1176472 t^{2} x^{2}-254.1176470 t x^{4} \\
& +1129.411764 x^{4}+7.058823529 t^{4}+0.4656862748 t x^{8} \\
& +798.6147174 t x^{3}-8.8180375507 t^{2} x^{5}-6.705882349 t^{3} x^{2} \\
& -1.455882353 t^{3} x^{4}-0.2911764705 t^{4} x^{2}-0.2870021640 t^{4} x^{3} \\
& +0.01360294119 t^{4} x^{4}+28.28427124 t x^{5}-0.1176470588 t^{2} x^{6} \\
& +0.5407287154 t^{3} x^{3}+0.01143849208 t^{3} x^{5}+39.93073589 t^{3} x \\
& -0.4991341962 t^{4} x+0.0004939348840 t^{6} x^{3}+0.07916666674 t^{3} x^{6} \\
& +0.02066727542 t^{4} x^{5}+0.002683823532 t^{5} x^{4}+0.8318903301 x^{9} \\
& +0.2010401634 t^{2} x^{7}-16.63780660 x^{7}-0.008823529412 t^{6} \\
& +0.0008823529411 t^{6} x^{2}+0.0003119588732 t^{6} x+0.1764705882 t^{5} \\
& \left.+0.001585790946 t^{5} x^{3}-0.005735294120 t^{5} x^{2}-0.02495670995 t^{5} x\right),
\end{aligned}
$$

$q(x, t)$

$$
\begin{array}{r}
=\left|\begin{array}{ccc}
1 & 1 & 1 \\
-0.6510416666 \times 10^{-5} t^{2} x^{4} & \mathcal{G} & 0.00007812500000 t^{2} x^{2} \\
-0.9223090280 \times 10^{-5} t x^{6} & -0.6510416666 \times 10^{-5} t^{2} x^{4} & \mathcal{G}
\end{array}\right| \\
=0.6004616067 \times 10^{-10} x^{6}\left(\begin{array}{l}
14.11764706 t^{4}+2.11764706 t^{3} x^{2}+0.7058823527 t^{4} x^{2} \\
+
\end{array}\right. \\
+208.2352942 t^{2} x^{2}+79.86147180 t^{3} x+1.996536794 t^{4} x \\
\left.+56.56854248 t x^{5}+20.00000001 t^{2} x^{4}+2.828427126 t^{3} x^{3}\right),
\end{array}
$$


where $\mathcal{G}$ denotes $0.0003682847818 x^{5}+0.0001302088884 t x^{4}+0.00001841423910 t^{2} x^{3}$. So the multivariate Padé approximation is of order $(5,2)$ for $(4.35)$, that is,

$$
\begin{aligned}
{[5,2]_{(x, t)}=} & -399.3073584 x^{5}+1.529411773 t^{2} x^{4}-25.29411764 t x^{6}-49.91341983 t^{2} x^{3} \\
& +254.1176472 t^{2} x^{2}-254.1176470 t x^{4}+1129.411764 x^{4}+7.058823529 t^{4} \\
& +0.4656862748 t x^{8}+798.6147174 t x^{3}-8.8180375507 t^{2} x^{5}-6.705882349 t^{3} x^{2} \\
& -1.455882353 t^{3} x^{4}-0.2911764705 t^{4} x^{2}-0.2870021640 t^{4} x^{3}+0.01360294119 t^{4} x^{4} \\
& +28.28427124 t x^{5}-0.1176470588 t^{2} x^{6}+0.5407287154 t^{3} x^{3}+0.01143849208 t^{3} x^{5} \\
& +39.93073589 t^{3} x-0.4991341962 t^{4} x+0.0004939348840 t^{6} x^{3}+0.07916666674 t^{3} x^{6} \\
& +0.02066727542 t^{4} x^{5}+0.002683823532 t^{5} x^{4}+0.8318903301 x^{9}+0.2010401634 t^{2} x^{7} \\
& -16.63780660 x^{7}-0.008823529412 t^{6}+0.0008823529411 t^{6} x^{2}+0.0003119588732 t^{6} x \\
& +0.1764705882 t^{5}+0.001585790946 t^{5} x^{3}-0.005735294120 t^{5} x^{2} \\
& \left.-0.02495670995 t^{5} x\right) \\
& /\left(14.11764706 t^{4}+2.11764706 t^{3} x^{2}+0.7058823527 t^{4} x^{2}\right. \\
& +508.2352942 t^{2} x^{2}+79.86147180 t^{3} x+1.996536794 t^{4} x+2258.823528 x^{4} \\
& +1597.229435 x^{3} t+39.93073587 t^{2} x^{3}+56.56854248 t x^{5}+20.00000001 t^{2} x^{4} \\
& \left.+2.828427126 t^{3} x^{3}\right) .
\end{aligned}
$$

We have the generalized differential transform method solution for the time-fractional Fitzhugh-Nagumo equation (4.27) (when $\alpha=0.50$ and $\mu=0.45$ ) as

$$
\begin{aligned}
u(x, t)= & 0.5000000000-0.1767766952 x-0.007365695635 x^{3}+0.0003682847818 x^{5} \\
+ & 0.05641895835\left(0.2500000000-0.03125000000 x^{2}+0.002604166667 x^{4}\right. \\
& \left.-0.0001844618056 x^{6}\right) t^{0.5} \\
- & 0.002500000000\left(0.5000000000-0.08838834762 x-0.06250000000 x^{2}\right. \\
= & \left.-0.01473139128 x^{3}+0.005208333333 x^{4}\right) t \\
& +0.01410473959 t^{0.5}-0.001763092448 t^{0.5} x^{2}+0.0001469243707 t^{0.5} x^{4} \\
& -0.00001040714293 t^{0.5} x^{6}-0.001250000000 t+0.0002209708690 t x \\
& +0.0001562500000 t x^{2}+0.00003682847820 t x^{3}-0.00001302083333 t x^{4} .
\end{aligned}
$$


For simplicity, let $t^{1 / 2}=a$, then

$$
\begin{aligned}
u(x, a)= & 0.5000000000-0.1767766952 x-0.007365695635 x^{3}+0.0003682847818 x^{5} \\
& +0.01410473959 a-0.001763092448 a x^{2}+0.0001469243707 a x^{4} \\
& -0.00001040714293 a x^{6}-0.001250000000 a^{2}+0.0002209708690 a^{2} x \\
& +0.0001562500000 a^{2} x^{2}+0.00003682847820 a^{2} x^{3}-0.00001302083333 a^{2} x^{4},
\end{aligned}
$$

and let

$$
\begin{aligned}
S= & 0.5000000000-0.1767766952 x-0.007365695635 x^{3}+0.0003682847818 x^{5} \\
& +0.01410473959 a-0.001763092448 a x^{2}+0.0001469243707 a x^{4}-0.001250000000 a^{2} \\
& +0.0002209708690 a^{2} x+0.0001562500000 a^{2} x^{2}+0.00003682847820 a^{2} x^{3} \\
T= & 0.5000000000-0.1767766952 x-0.007365695635 x^{3}+0.01410473959 a \\
& -0.001763092448 a x^{2} 0.001250000000 a^{2}+0.0002209708690 a^{2} x+0.0001562500000 a^{2} x^{2}, \\
V= & 0.5000000000-0.1767766952 x-0.007365695635 x^{3}+0.01410473959 a \\
& -0.001763092448 a x^{2}-0.001250000000 a^{2}+0.0002209708690 a^{2} x .
\end{aligned}
$$

Then, using (2.5) to calculate the multivariate Padé equations for (4.42), we get,

$$
\begin{aligned}
& p(x, a) \\
& =\left|\begin{array}{ccc}
S & T & V \\
-0.00001302083333 a^{2} x^{4} & \mathscr{d e} & 0.0001562500000 a^{2} x^{2} \\
-0.00001040714293 a x^{6} & -0.00001302083333 a^{2} x^{4} & \not d
\end{array}\right| \\
& =0.1355096735 \times 10^{-9} x^{6}\left(-176.9384663 x^{5}+500.4575575 x^{4}+14.14213562 a x^{5}\right. \\
& -0.1042619911 a^{2} x^{6}-127.0588234 a x^{4}-12.64705882 a x^{6} \\
& +179.7418057 a^{2} x^{2}-34.59022553 a^{2} x^{3}-1.864967528 a^{2} x^{4} \\
& +0.01143849208 a^{3} x^{5}+39.93073588 a^{3} x-1.527651730 a^{4} x \\
& +0.5407287176 a^{3} x^{3}+12.511438894 a^{4}+0.2328431370 a x^{8} \\
& +0.3686218050 x^{9}-7.372436097 x^{7}-5.430474480 a^{2} x^{5} \\
& -7.988400797 a^{3} x^{2}-1.386412603 a^{3} x^{4}-0.4194856542 a^{4} x^{2} \\
& -0.4711921255 a^{4} x^{3}+0.02008511870 a^{4} x^{4}+399.3073586 a x^{3} \\
& +0.0011750953574 a^{6} x^{3}+0.07716273155 a^{3} x^{6}+0.02800876865 a^{4} x^{5} \\
& +0.005367647059 a^{5} x^{4}+0.1536097286 a^{2} x^{7}-0.04991341984 a^{5} x \\
& -0.03127859737 a^{6}+0.003127859737 a^{6} x^{2}+0.01147058823 a^{5} x^{2} \\
& \left.+0.3529411765 a^{5}+0.003171581881 a^{5} x^{3}-0.01147058823 a^{5} x^{2}\right),
\end{aligned}
$$




$$
\begin{aligned}
& q(x, a) \\
& =\left|\begin{array}{ccc}
1 & 1 & 1 \\
-0.00001302083333 a^{2} x^{4} & \mathscr{L} & 0.0001562500000 a^{2} x^{2} \\
-0.00001040714293 a x^{6} & -0.00001302083333 a^{2} x^{4} & \mathscr{d}
\end{array}\right| \\
& =0.1355096735 \times 10^{-9} x^{6}\left(25.02287789 a^{4}+2.11764705 a^{3} x^{2}+1.2511438894 a^{4} x^{2}\right. \\
& +359.4836114 a^{2} x^{2}+79.86147175 a^{3} x+3.538769329 a^{4} x \\
& +1000.915115 x^{4}+798.6147171 x^{3} a+35.38769327 a^{2} x^{3} \\
& \left.+28.28427125 x^{5} a+11.28379167 a^{2} x^{4}+2.828427126 a^{3} x^{3}\right),
\end{aligned}
$$

where $\mathscr{H}$ denotes $0.0003682847818 x^{5}+0.0001469243707 a x^{4}+0.00003682847820 a^{2} x^{3}$, recalling that $t^{1 / 2}=a$, we get multivariate Padé approximation of order $(5,2)$ for $(4.40)$, that is,

$$
\begin{aligned}
{[5,2]_{(x, t)}=(-} & 176.9384663 x^{5}+500.4575575 x^{4}+14.14213562 \sqrt{t} x^{5} \\
- & 0.1042619911 t x^{6}-127.0588234 \sqrt{t} x^{4}-12.64705882 \sqrt{t} x^{6}+179.7418057 t x^{2} \\
& -34.59022553 t x^{3}-1.864967528 t x^{4}+0.01143849208 t^{3 / 2} x^{5}+39.93073588 t^{3 / 2} x \\
- & 1.527651730 t^{2} x+0.5407287176 t^{3 / 2} x^{3}+12.511438894 t^{2}+0.2328431370 \sqrt{t} x^{8} \\
+ & 0.3686218050 x^{9}-7.372436097 x^{7}-5.430474480 t x^{5}-7.988400797 t^{3 / 2} x^{2} \\
- & 1.386412603 t^{3 / 2} x^{4}-0.4194856542 t^{2} x^{2}-0.4711921255 t^{2} x^{3}+0.02008511870 t^{2} x^{4} \\
+ & 399.3073586 \sqrt{t} x^{3}+0.0011750953574 t^{3} x^{3}+0.07716273155 t^{3 / 2} x^{6} \\
+ & 0.02800876865 t^{2} x^{2}+0.005367647059 t^{5 / 2} x^{4}+0.1536097286 t x^{7} \\
- & 0.04991341984 t^{5 / 2} x-0.03127859737 t^{3}+0.003127859737 t^{3} x^{2} \\
+ & 0.01147058823 t^{5 / 2} x^{2}+0.3529411765 t^{5 / 2} \\
+ & \left.0.003171581881 t^{5 / 2} x^{3}-0.01147058823 t^{5 / 2} x^{2}\right) \\
& /\left(25.02287789 t^{2}+2.11764705 t^{3 / 2} x^{2}+1.2511438894 t^{2} x^{2}+359.4836114 t x^{2}\right. \\
& +79.86147175 t^{3 / 2} x+3.538769329 t^{2} x+1000.915115 x^{4}+798.6147171 x^{3} \sqrt{t} \\
& \left.+35.38769327 t x^{3}+28.28427125 x^{5} \sqrt{t}+11.28379167 t x^{4}+2.828427126 t^{3 / 2} x^{3}\right) .
\end{aligned}
$$


We have the generalized differential transform method solution for the time-fractional Fitzhugh-Nagumo equation (4.27) (when $\alpha=0.75$ and $\mu=0.45$ ) as

$$
\begin{aligned}
u(x, t)= & 0.5000000000-0.1767766952 x-0.007365695635 x^{3}+0.0003682847818 x^{5} \\
& +0.05440326260\left(0.2500000000-0.03125000000 x^{2}\right. \\
& \left.+0.002604166667 x^{4}-0.0001844618056 x^{6}\right) t^{0.75} \\
& -0.001880631946\left(0.5000000000-0.08838834762 x-0.06250000000 x^{2}\right. \\
= & 0.5000000000-0.1767766952 x-0.007365695635 x^{3}+0.0003682847818 x^{5} \\
& +0.01360081565 t^{0.75}-0.001700101956 t^{0.75} x^{2}+0.0001416751630 t^{0.75} x^{4} \\
& -0.00001003532405 t^{0.75} x^{6}-0.0009403159730 t^{1.50}+0.0001662259502 t^{1.50} x \\
& +0.0001175394966 t^{1.50} x^{2}+0.00002770432505 t^{1.50} x^{3}-0.9794958051 \times 10^{-5} t^{1.50} x^{4} .
\end{aligned}
$$

For simplicity, let $t^{1 / 4}=a$, then

$$
\begin{aligned}
u(x, a)= & 0.5000000000-0.1767766952 x-0.007365695635 x^{3}+0.0003682847818 x^{5} \\
& +0.01360081565 a^{3}-0.001700101956 a^{3} x^{2}+0.0001416751630 a^{3} x^{4} \\
& -0.00001003532405 a^{3} x^{6}-0.0009403159730 a^{6}+0.0001662259502 a^{6} x \\
& +0.0001175394966 a^{6} x^{2}+0.00002770432505 a^{6} x^{3}-0.9794958051 \times 10^{-5} a^{6} x^{4}
\end{aligned}
$$

and let

$$
\begin{aligned}
Y= & 0.5000000000-0.1767766952 x-0.007365695635 x^{3}+0.0003682847818 x^{5} \\
& +0.01360081565 a^{3}-0.001700101956 a^{3} x^{2}+0.0001416751630 a^{3} x^{4}-0.0009403159730 a^{6} \\
& +0.0001662259502 a^{6} x+0.0001175394966 a^{6} x^{2} \\
Z= & 0.5000000000-0.1767766952 x-0.007365695635 x^{3}+0.0003682847818 x^{5} \\
& +0.01360081565 a^{3}-0.001700101956 a^{3} x^{2}+0.0001416751630 a^{3} x^{4}-0.0009403159730 a^{6} \\
& +0.0001662259502 a^{6} x \\
I= & 0.5000000000-0.1767766952 x-0.007365695635 x^{3}+0.0003682847818 x^{5} \\
& +0.01360081565 a^{3}-0.001700101956 a^{3} x^{2}-0.0009403159730 a^{6} .
\end{aligned}
$$


Then, using (2.5) to calculate the multivariate Padé equations for (4.48), we get

$$
\begin{aligned}
& p(x, a)=\left|\begin{array}{ccc}
Y & Z & I \\
\supset & 0.0001175394966 a^{6} x^{2} & \partial \\
-0.9794958051 \times 10^{-5} a^{6} x^{4} & \partial & 0.0001175394966 a^{6} x^{2}
\end{array}\right| \\
& =-0.9794958051 \times 10^{-5} x^{4}\left(-0.00007257591929 x^{6}-0.514079428 \times 10^{-5} x^{8}\right. \\
& +0.2227383885 \times 10^{-7} x^{11}+0.00002462745270 a^{3} x^{5} \\
& +0.0001152063889 a^{3} x^{3}-0.3786552603 \times 10^{-8} x^{13} \\
& +0.2886689512 \times 10^{-5} x^{9}+0.00002565946232 x^{7} \\
& -0.00003010597218 a^{3} x^{4}+0.1477000393 \times 10^{-5} a^{6} x^{5} \\
& -0.2263460486 \times 10^{-6} a^{3} x^{8}+0.2840147227 \times 10^{-6} a^{6} x^{6} \\
& +0.7826520955 \times 10^{-8} a^{3} x^{9}-0.4382354311 \times 10^{-8} a^{6} x^{8} \\
& -0.1065755353 \times 10^{-5} a^{9} x^{2}-0.1064464005 \times 10^{-5} a^{9} x^{3} \\
& +0.6782425517 \times 10^{-5} a^{9} x-0.00001278906419 a^{9} \\
& -0.1601429235 \times 10^{-6} a^{6} x^{7}+0.00004469028926 a^{6} x^{3} \\
& -0.00001370665784 a^{3} x^{6}-0.3384074604 \times 10^{-5} a^{6} x^{4} \\
& -0.1565304191 \times 10^{-6} a^{3} x^{7}+0.4788518010 \times 10^{-7} a^{9} x^{5} \\
& +0.2181467984 \times 10^{-6} a^{9} x^{4}+0.1782226356 \times 10^{-7} a^{3} x^{10} \\
& +0.884194129 \times 10^{-6} a^{12}+0.1565760439 \times 10^{-6} a^{12} x^{2} \\
& -0.6252196644 \times 10^{-5} a^{12} x-0.0004701579863 a^{6} \\
& \left.-0.0001861042030 a^{6} x^{2}+0.0004155648754 a^{6} x\right) a^{6} \\
& q(x, a)=\left|\begin{array}{ccc}
1 & 1 & 1 \\
\jmath & 0.0001175394966 a^{6} x^{2} & \partial \\
-0.9794958051 \times 10^{-5} a^{6} x^{4} & \jmath & 0.0001175394966 a^{6} x^{2}
\end{array}\right| \\
& =-0.9794958051 \times 10^{-5} x^{4}\left(0.0009403159722 a^{6}-0.0002304127778 a^{3} x^{3}\right. \\
& -0.0004986778508 a^{6} x+0.0001451518386 x^{6} \\
& -0.0000212512744 a^{3} x^{4}-0.00005676836553 a^{3} x^{5} \\
& \left.+0.00001028158857 x^{8}+0.0001958991611 a^{6} x^{3}\right) a^{6},
\end{aligned}
$$


where $\int$ denotes $0.00002770432505 a^{6} x^{3}-0.00001003532405 a^{3} x^{6}$, and 2 denotes $0.0001662259502 a^{6} x+0.0001416751630 a^{3} x^{4}$; recalling that $t^{1 / 4}=a$ we get multivariate Padé approximation of order $(8,2)$ for $(4.46)$, that is,

$$
\begin{aligned}
{[8,2]_{(x, t)} } & \\
=-( & -0.00007257591929 x^{6}-0.514079428 \times 10^{-5} x^{8}+0.2227383885 \times 10^{-7} x^{11} \\
& +0.00002462745270 t^{3 / 4} x^{5}+0.0001152063889 t^{3 / 4} x^{3}-0.3786552603 \times 10^{-8} x^{13} \\
& +0.2886689512 \times 10^{-5} x^{9}+0.00002565946232 x^{7}-0.00003010597218 t^{3 / 4} x^{4} \\
+ & 0.1477000393 \times 10^{-5} t^{3 / 2} x^{5}-0.2263460486 \times 10^{-6} t^{3 / 4} x^{8}+0.2840147227 \times 10^{-6} t^{3 / 2} x^{6} \\
+ & 0.7826520955 \times 10^{-8} t^{3 / 4} x^{9}-0.4382354311 \times 10^{-8} t^{3 / 2} x^{8}-0.1065755353 \times 10^{-5} t^{9 / 4} x^{2} \\
& -0.1064464005 \times 10^{-5} t^{9 / 4} x^{3}+0.6782425517 \times 10^{-5} t^{9 / 4} x-0.00001278906419 t^{9 / 4} \\
& -0.1601429235 \times 10^{-6} t^{3 / 2} x^{7}+0.00004469028926 t^{3 / 2} x^{3}-0.00001370665784 t^{3 / 4} x^{6} \\
& -0.3384074604 \times 10^{-5} t^{3 / 2} x^{4}-0.1565304191 \times 10^{-6} t^{3 / 4} x^{7}+0.4788518010 \times 10^{-7} t^{9 / 4} x^{5} \\
+ & 0.2181467984 \times 10^{-6} t^{9 / 4} x^{4}+0.1782226356 \times 10^{-7} t^{3 / 4} x^{10}+0.884194129 \times 10^{-6} t^{3} \\
+ & 0.1565760439 \times 10^{-6} t^{3} x^{2}-0.6252196644 \times 10^{-5} t^{3} x-0.0004701579863 t^{3 / 2} \\
& \left.-0.0001861042030 t^{3 / 2} x^{2}+0.0004155648754 t^{3 / 2} x\right) \\
& /\left(0.0009403159722 t^{3 / 2}-0.0002304127778 t^{3 / 4} x^{3}\right. \\
& -0.0004986778508 t^{3 / 2} x+0.0001451518386 x^{6} \\
& -0.0000212512744 t^{3 / 4} x^{4}-0.00005676836553 t^{3 / 4} x^{5} \\
& \left.+0.00001028158857 x^{8}+0.0001958991611 t^{3 / 2} x^{3}\right) .
\end{aligned}
$$

As it is presented above, we obtained multivariate Padé approximations of the generalized differential transform method solution of the time-fractional Fitzhugh-Nagumo equation (4.27) for values of $\alpha=1.0, \alpha=0.50$, and $\alpha=0.75$. Table 3 shows the approximate solutions for (4.27) obtained for different values of $\alpha$ using the generalized differential transform method (GDTM) and the multivariate Padé approximation (MPA). The values of $\alpha=1.0$ are the only case for which we know the exact solution $u(x, t)=$ $1 /\left(1+e^{(1 / \sqrt{2})(x+((1-2 \mu) / \sqrt{2}) t)}\right)$, and the results of multivariate Padé approximation (MPA) are in excellent agreement with the exact solution and those obtained by the generalized differential transform method (GDTM). 


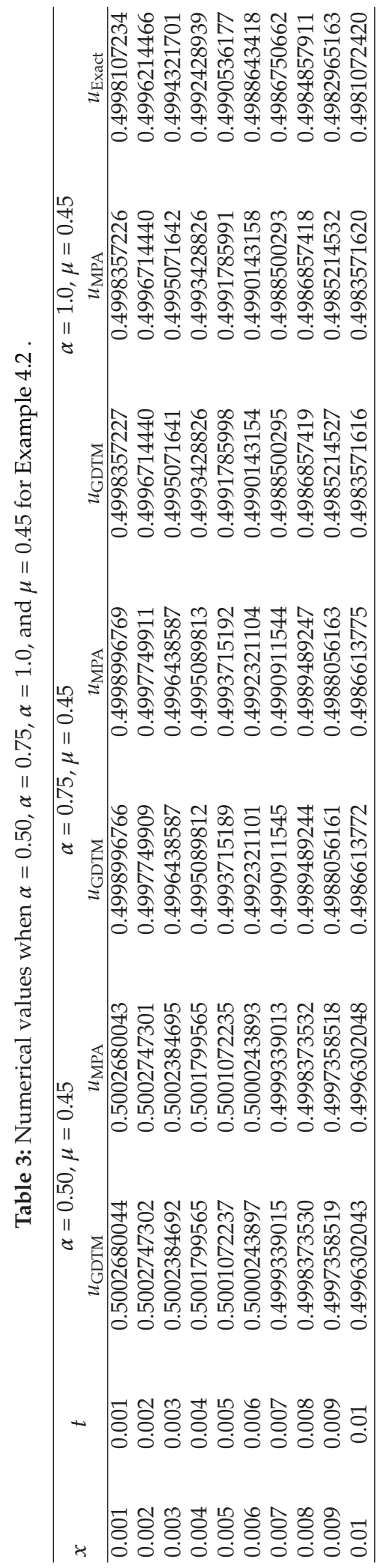




\section{Conclusion}

By comparison with the generalized differential transform method (GDTM), the fundamental goal of this work has been to construct an approximate solution for time-fractional reactiondiffusion equations by using multivariate Padé approximation. The goal has been achieved by using the multivariate Pade approximation (MPA) and the generalized differential transform method (GDTM). The present work shows the validity and great potential of the multivariate Padé approximation for solving time-fractional reaction-diffusion equations from the numerical results. For the values of $\alpha=1.0$ in Example 4.1 and for the values of $\alpha=1.0$ in Example 4.2, numerical results obtained using the multivariate Padé approximation (MPA) and the generalized differential transform method (GDTM) are in excellent agreement with exact solutions and each other. For the values of $\alpha=0.50, \alpha=0.75$, in Example 4.1 and for the values of $\alpha=0.50, \alpha=0.75$ in Example 4.2, numerical results show that the results of multivariate Padé approximation are in excellent agreement with those results obtained by the generalized differential transform method (GDTM). The basic idea described in this paper is expected to be further employed to solve other similar problems in fractional calculus.

\section{References}

[1] I. Podlubny, Fractional Differential Equations, Academic Press, San Diego, Calif, USA, 1990.

[2] I. S. Jesus and J. A. Tenreiro Machado, "Implementation of fractional-order electromagnetic potential through a genetic algorithm," Communications in Nonlinear Science and Numerical Simulation, vol. 14, no. 5, pp. 838-843, 2009.

[3] F. C. Meral, T. J. Royston, and R. Magin, "Fractional calculus in viscoelasticity: an experimental study," Communications in Nonlinear Science and Numerical Simulation, vol. 15, no. 4, pp. 939-945, 2010.

[4] A. C. Galucio, J. F. Deu, and R. Ohayon, "Finite element formulation of viscoelastic sandwich beams using fractional derivative operators," Computational Mechanics, vol. 33, no. 4, pp. 282-291, 2004.

[5] K. Seki, M. Wojcik, and M. Tachiya, "Fractional reaction-diffusion equation," Journal of Chemical Physics, vol. 119, pp. 2165-2174, 2003.

[6] B. I. Henry and S. L. Wearne, "Fractional reaction-diffusion," Physica A, vol. 276, no. 3-4, pp. 448-455, 2000.

[7] B. Baeumer, M. Kovács, and M. M. Meerschaert, "Numerical solutions for fractional reaction-diffusion equations," Computers \& Mathematics with Applications, vol. 55, no. 10, pp. 2212-2226, 2008.

[8] S. B. Yuste, L. Acedo, and K. Lindenberg, "Reaction front in an $A+B \rightarrow C$ reaction-subdiffusion process," Physical Review E, vol. 69, no. 3, part 2, Article ID 036126, 2004.

[9] R. A. Fisher, "The wave of advance of advantageous genes," Annals of Human Genetics, vol. 7, no. 4, pp. 335-369, 1937.

[10] W. Malfliet, "Solitary wave solutions of nonlinear wave equations," American Journal of Physics, vol. 60, no. 7, pp. 650-654, 1992.

[11] D. A. Frank, Diffusion and Heat Exchange in Chemical Kinetics, Princeton University Press, Princeton, NJ, USA, 1955.

[12] D. J. Aronson and H. F. Weinberg, Nonlinear Diffusion in Population Genetics Combustion and Never Pulse Propagation, Springer, New York, NY, USA, 1988.

[13] J. Canosa, "Diffusion in nonlinear multiplication media," Journal of Mathematical Physics, vol. 10, no. 10, pp. $1862-1869,1969$.

[14] H. C. Tuckwell, Introduction to Theoretical Neurobiology, : Cambridge University Press, Cambridge, UK, 1988.

[15] M. D. Bramson, "Maximal displacement of branching Brownian motion," Communications on Pure and Applied Mathematics, vol. 31, no. 5, pp. 531-581, 1978.

[16] R. Fitzhugh, "Impulse and physiological states in theoretical models of nerve membrane," Biophysical Journal, vol. 1, no. 6, pp. 445-466, 1961.

[17] J. S. Nagumo, S. Arimoto, and S. Yoshizawa, "An active pulse transmission line simulating nerve axon," Proceedings of the IRE, vol. 50, pp. 2061-2070, 1962. 
[18] D. G. Aronson and H. F. Weinberger, "Multidimensional nonlinear diffusion arising in population genetics," Advances in Mathematics, vol. 30, no. 1, pp. 33-76, 1978.

[19] Z. Odibat, S. Momani, and V. S. Erturk, "Generalized differential transform method for solving a space- and time-fractional diffusion-wave equation," Physics Letters A, vol. 370, no. 5-6, pp. 379-387, 2007.

[20] S. Momani, Z. Odibat, and V. S. Erturk, "Generalized differential transform method: application to differential equations of fractional order," Applied Mathematics and Computation, vol. 197, no. 2, pp. 467-477, 2008.

[21] Z. Odibat and S. Momani, "A generalized differential transform method for linear partial differential equations of fractional order," Applied Mathematics Letters, vol. 21, no. 2, pp. 194-199, 2008.

[22] F. Ayaz, "Solutions of the system of differential equations by differential transform method," Applied Mathematics and Computation, vol. 147, no. 2, pp. 547-567, 2004.

[23] A. Arikoglu and I. Ozkol, "Solution of boundary value problems for integro-differential equations by using differential transform method," Applied Mathematics and Computation, vol. 168, no. 2, pp. 1145-1158, 2005.

[24] N. Bildik, A. Konuralp, F. Orakçı Bek, and S. Küçükarslan, "Solution of different type of the partial differential equation by differential transform method and Adomian's decomposition method," Applied Mathematics and Computation, vol. 172, no. 1, pp. 551-567, 2006.

[25] I. H. Hassan, "Comparison differential transformation technique with Adomian decomposition method for linear and nonlinear initial value problems," Chaos, Solitons and Fractals, vol. 36, no. 1, pp. 53-65, 2008.

[26] Z. M. Odibat, C. Bertelle, M. A. Aziz-Alaoui, and G. H. E. Duchamp, "A multi-step differential transform method and application to non-chaotic or chaotic systems," Computers $\mathcal{E}$ Mathematics with Applications, vol. 59, no. 4, pp. 1462-1472, 2010.

[27] S. Z. Rida, A. M. A. El-Sayed, and A. A. M. Arafa, "On the solutions of time-fractional reactiondiffusion equations," Communications in Nonlinear Science and Numerical Simulation, vol. 15, no. 12, pp. 3847-3854, 2010.

[28] S. Momani and R. Qaralleh, "Numerical approximations and Padé approximants for a fractional population growth model," Applied Mathematical Modelling, vol. 31, no. 9, pp. 1907-1914, 2007.

[29] S. Momani and N. Shawagfeh, "Decomposition method for solving fractional Riccati differential equations," Applied Mathematics and Computation, vol. 182, no. 2, pp. 1083-1092, 2006.

[30] A. Cuyt and L. Wuytack, Nonlinear Methods in Numerical Analysis, Elsevier Science, Amsterdam, The Netherland, 1987.

[31] P. Guillaume and A. Huard, "Multivariate Padé approximation," Journal of Computational and Applied Mathematics, vol. 121, no. 1-2, pp. 197-219, 2000.

[32] A. Cuyt, "A review of multivariate Padé approximation theory," Journal of Computational and Applied Mathematics, vol. 12, pp. 221-232, 1985.

[33] P. Zhou, "Explicit construction of multivariate Padé approximants," Journal of Computational and Applied Mathematics, vol. 79, no. 1, pp. 1-17, 1997.

[34] A. Cuyt, L. Wuytack, and H. Werner, "On the continuity of the multivariate Padé operator," Journal of Computational and Applied Mathematics, vol. 11, no. 1, pp. 95-102, 1984.

[35] A. Cuyt, "How well can the concept of Padé approximant be generalized to the multivariate case?" Journal of Computational and Applied Mathematics, vol. 105, no. 1-2, pp. 25-50, 1999.

[36] P. Guillaume, A. Huard, and V. Robin, "Generalized multivariate Padé approximants," Journal of Approximation Theory, vol. 95, no. 2, pp. 203-214, 1998.

[37] A. A. M. Cuyt, "Multivariate Padé-approximants," Journal of Mathematical Analysis and Applications, vol. 96, no. 1, pp. 283-293, 1983.

[38] J. Abouir, A. Cuyt, P. Gonzalez-Vera, and R. Orive, "On the convergence of general order multivariate Padé-type approximants," Journal of Approximation Theory, vol. 86, no. 2, pp. 216-228, 1996.

[39] P. Zhou, "Multivariate Padé approximants associated with functional relations," Journal of Approximation Theory, vol. 93, no. 2, pp. 201-230, 1998.

[40] V. Turut, E. Çelik, and M. Yiğider, "Multivariate Padé approximation for solving partial differential equations (PDE)," International Journal for Numerical Methods in Fluids, vol. 66, no. 9, pp. 1159-1173, 2011.

[41] M. Caputo, "Linear models of dissipation whose $Q$ is almost frequency independent. Part II," Geophysical Journal International, vol. 13, no. 5, pp. 529-539, 1967. 


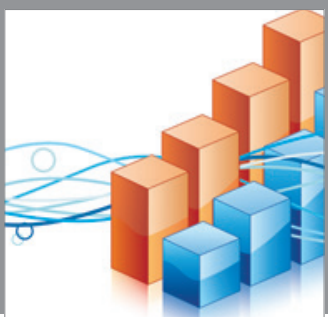

Advances in

Operations Research

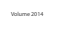

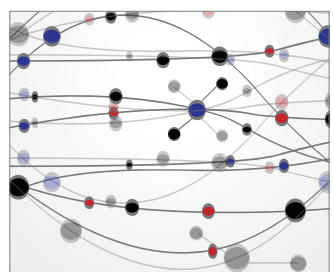

\section{The Scientific} World Journal
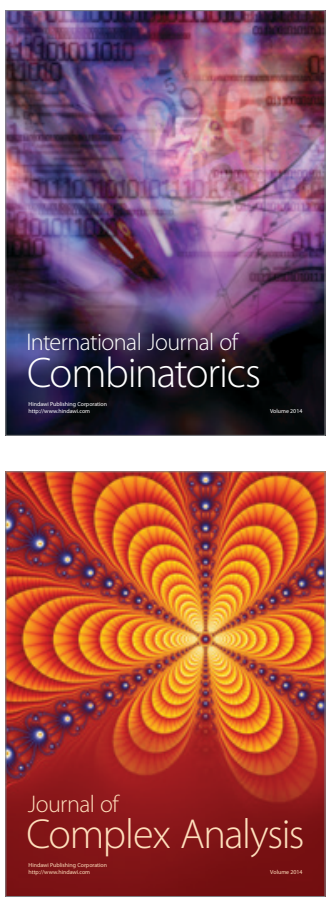

International Journal of

Mathematics and

Mathematical

Sciences
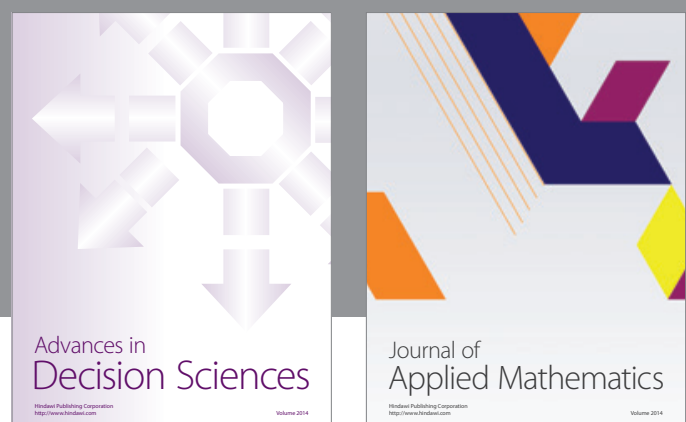

Journal of

Applied Mathematics
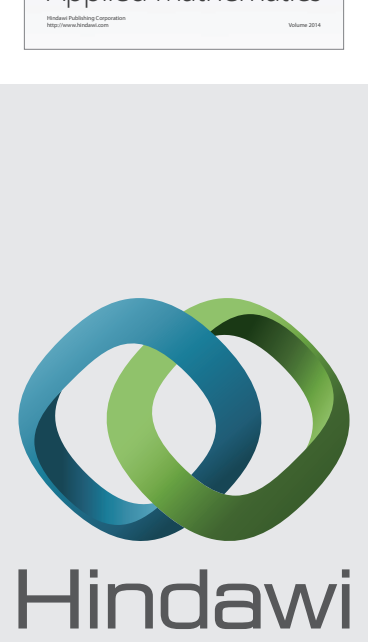

Submit your manuscripts at http://www.hindawi.com
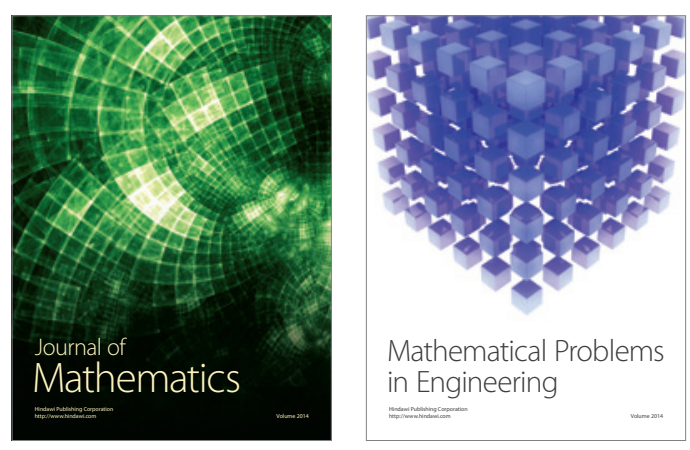

Mathematical Problems in Engineering
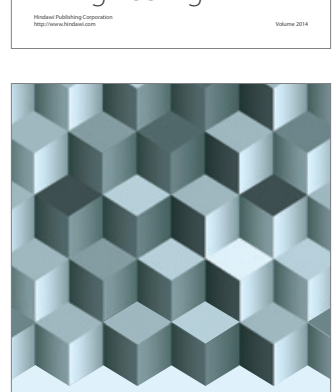

Journal of

Function Spaces
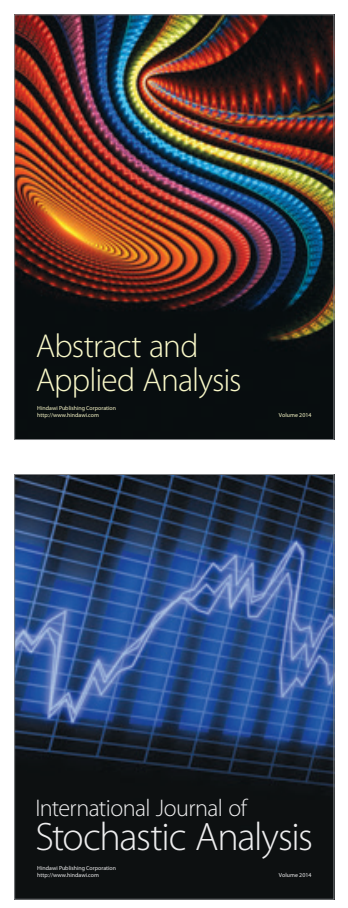

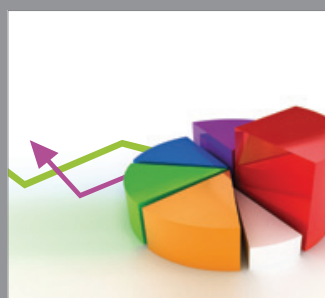

ournal of

Probability and Statistics

Promensencen
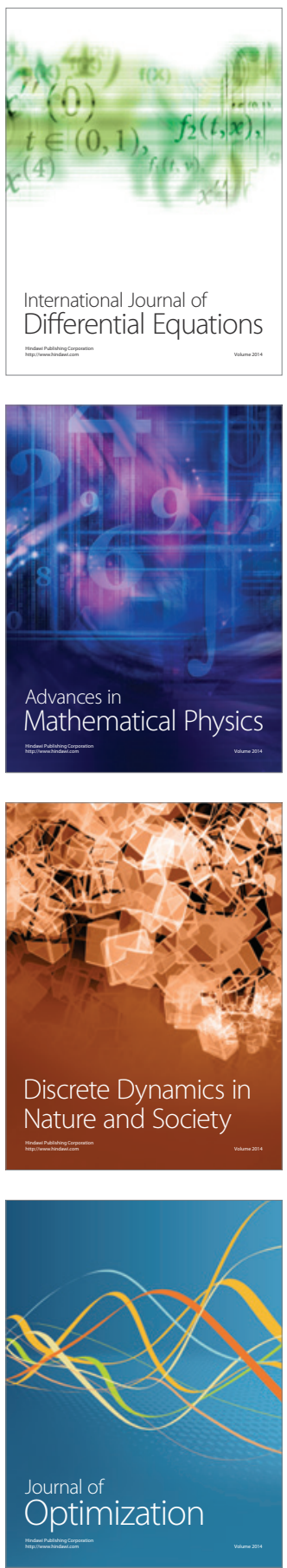